\title{
Nucleation and growth of a single suspended protein crystal by merging phase separated droplets
}

\author{
D. C. Yin*, Z. Q. Wu, Y. M. Liu \\ School of Life Sciences, Key Laboratory for Space Bioscience and Biotechnology, Northwestern Polytechnical University, Xi'an \\ 710072, Shaanxi, PR China \\ yindc@nwpu.edu.cn
}

Protein crystallization is an ideal theoretical model for studying nucleation and growth of crystals due to features like slow kinetics. In the research field of crystal nucleation, there are several important theories describing the nucleation process. Among the theoreis, nucleation from dense liquid droplets after a process called liquid-liquid phase separation (LLPS) is widely accepted. According to this theory, the number and distribution of the phase separated droplets can determine the number, size and distribution of the final crystals in the solution. Here in this report, we will present our effort to obtain a single, suspended crystal in the crystallization solution through manipulation of the phase separated droplets. It is known that gradient magnetic field can exert magnetic force on the objects in the field so that gradient magnetic field can be fully utilized in protein crystallization [1-3]. By using a large gradient magnetic field we can merge the phase separated droplets in the solution non-contactly. The merged dense liquid droplet will be the location of protein crystallization and a single suspended crystal can be thus obtained. Figure 1 shows an example of suspened lysozyme crystal grown in a superconducting magnet. The results show that crystallization can be controlled via manipulation of the phase-separated droplets. Further, this study can also provide a strong support for the two-step nucleation theory.

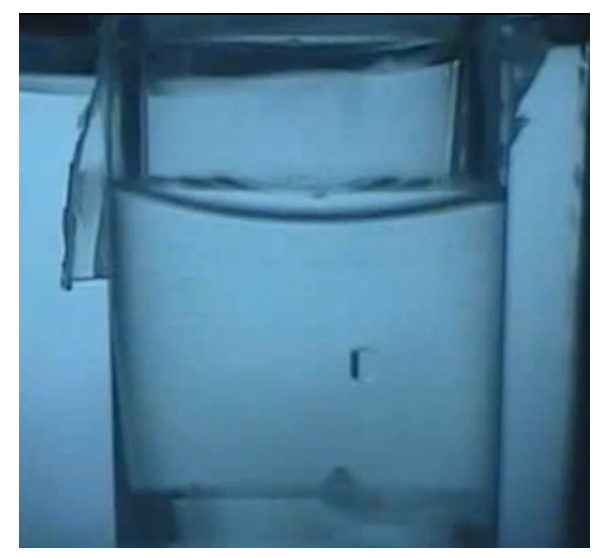

Figure 1. A single suspended lysozyme crystal grown by merging the phase separated droplets. The diameter of the container is $8 \mathrm{~mm}$.

[1] Pareja-Rivera, C., Cuellar-Cruz, M., Esturau-Escofet, N., Demitri, N., Polentarutti, M., Stojanoff, V. \& Moreno, A. (2017), Cryst. Growth Des., 17, 135-145.

[2] Ryu, S. Y., Oh, I. H., Cho, S. J., Kim, S. A. \& Song, H. K. (2020), Crystals, 10, 821.

[3] Yin, D. C. (2015), Prog. Cryst. Growth Charact. Mater. 61, 1-26.

Keywords: protein crystallization; single crystal; suspended crystal; levitation; magnetic field; liquid-liquid phase separation Acknowledgements: the Innovation Capability Support Program of Shaanxi (Program No. 2020TD-042). 